\title{
Information Technologies for the Prevention of Medication Errors
}

\author{
Pascal Bonnabry*
}

\begin{abstract}
Significant efforts are currently under way to improve the safety of the medication process in hospitals. Among other solutions, new developments involving information technologies (IT) have attracted growing attention, as they have the potential to attenuate a leading cause of error: human failure. IT interventions focus on the three major steps of drug use: prescription (computerized physician order entry, CPOE), dispensing (automated dispensing systems, ADS) and administration (scanning barcodes or RFID tags). Although these technologies are used more and more in hospitals, only few robust data are actually available to measure their real impact on patient safety. CPOE is the best studied, followed by ADS and scanning technologies. More data are necessary to demonstrate their usefulness or to generalize results obtained in selected environments. An increased use of IT in future years seems to be unavoidable, but its implementation will be a real challenge, since cultural, human, technical, and ergonomic aspects will have to be taken into consideration to avoid failure. Moreover, these technologies can also potentially induce new risks, e.g. data entry errors, that need a careful consideration during implementation. In conclusion, IT has a huge potential to help reduce the occurrence of medication errors in hospitals, but the real positive impact and the induced risks need to be systematically and scientifically evaluated in various environments. In the Geneva university hospitals, some developments are under way to implement IT on pilot processes, with a concomitant evaluation by a systematic risk analysis method (FMECA).
\end{abstract}

Keywords: Automated dispensing systems - Computerized physician order entry · Information technologies · Medication administration record $\cdot$ Medication errors

\section{Introduction}

In the last few years, medical adverse events have received growing attention. In a wellcovered report published in 1999 and entitled 'To err is human: building a safer health system' [1], the Institute of Medicine, relying on three studies performed during the last decade in the USA, estimated that the number of deaths attributable to preventable medical errors was probably between 44'000 and 98'000 per year [2][3], and that

${ }^{*}$ Correspondence: PD Dr. P. Bonnabry Pharmacy

University Hospitals of Geneva (HUG)

Rue Micheli-du-Crest 24

$\mathrm{CH}-1211$ Geneva 14

Tel.: +4122 3823974

Fax: +4122382 3970

E-Mail: Pascal.Bonnabry@hcuge.ch

http://www.hcuge.ch/Pharmacie
7'000 Americans could die annually from medication errors alone [4]. Another welldesigned study prospectively measured a rate of 6.5 adverse drug events per 100 admissions, of which $28 \%$ were preventable, with errors occurring at the stage of ordering $(49 \%)$, transcription (11\%), dispensing (14\%) and administration (26\%) [5].

Although health care processes are very complex, they are actually strongly based on human reliability, which explains the high rate of adverse events. Indeed, the performance of highly trained operators has been shown to be associated with error rates in the range of $10^{-2}$ for dispensation [6][7] and even $10^{-1}$ for calculation [7][8]. Mechanisms leading to active failure are multiple and include slips, lapses, mistakes, and procedural violations [9]. More importantly, the organization of the working environment has a major influence on the operator's performance, determining the latent conditions more or less favorable to the occurrence of active errors. Only a few factors such as illumination [10], sound [11], workload [10] and task disruption [12] have been so far investigated and shown to influence the error rate.

Significant efforts are today under way to prevent human errors and improve patient safety and great hopes have been pinned on information technologies (IT). These developments attract great interest because they have the potential to suppress manual or cognitive steps linked to high error rates and because they target systems rather than individuals; a strategy that has been shown to be effective in reducing errors in other fields such as aviation industry [13].

\section{Information Technologies in the Medication Process}

Numerous interventions involving information technologies have been developed to improve the safety of three major steps of drug use in hospitals: prescription (computerized physician order entry), dispensation (automated dispensing systems) and administration (scanning barcodes or RFID tags)

Computerized physician order entry (CPOE) refers to a variety of computerbased systems that share the common features of automating the medication ordering process and that ensure standardized, legible, and complete orders [14]. Clinical decision support systems (CDSSs) are built into almost all CPOE systems to 
varying degrees, providing basic computerized advice regarding drug doses, routes, frequencies, as well as more sophisticated data such as drug allergy, drug-laboratory values, drug-drug interactions checks and guidelines. Results of American surveys [15][16] indicate that 4 to $15 \%$ of hospitals have an electronic medication order-entry system in place. This relatively low rate reflects the recent emergence of these tools and the complexity to set up them.

Automated dispensing systems are drug storage devices or cabinets that electronically dispense medications in a controlled fashion and track medication use. Centralized systems (e.g. Baxter ATC-212, Homerus, Swisslog Pillpick system) prepare and distribute individualized treatments from a central location in the hospital, whereas decentralized pharmacies (e.g. Pyxis Medstation, Omnicell Sure-Med) reside on nursing wards. They can be directly connected to an electronic prescription system or require a retranscription of a written or electronic order in the dispensing device. The decentralized pharmacies offer several aspects of interest for nurses, in particular immediate access to medications, thereby decreasing turnaround time, and improved tracking of narcotics dispensing [17]. They try to cumulate the advantages of unit dose system (individualization to reduce error rate) and ward stocks (rapid availability of drugs). In a 2002 national survey [18] of drug dispensing practices in the USA, an estimated $8 \%$ of hospitals used a robotic distribution system that automates the dispensing of unit doses within the centralized distribution system. A majority of hospitals (58\%) with decentralized distribution systems employed automated point-of-use dispensing devices.

Scanning technologies can contribute to a reduction in medication errors by ensuring the 'five rights' of medication administration: right patient, right drug, right dose, right route, and right time. By linking a scanning device with CPOE and/or automated dispensing software, the performance of these final checks can be improved. When ready to administer a medication, the nurse simply scans a barcode or a RFID tag on its own identification badge, the barcodes of medications to be administered, and then the patient's wristband barcode or RFID tag. As a by-product these systems provide a complete and accurate online electronic medication administration record (eMAR) by documenting precise administration data and making it easier for all caregivers to trace a patient's care over time. Actually, only $1.5 \%$ of hospitals use scanning technology in the administration process to verify the correct patient and the correct drug, but nearly two-thirds of hospitals use computer-generated MARs [19].

If we try to imagine what the medication system of the future could be, we will have physicians ordering online, supported by a clinical decision system. The orders will be sent electronically to a robot that will automatically fill the patient-cart or to a decentralized automated dispensing system manipulated by the nurses. All drugs, patients, and staff will have an electronic identification, making it possible to perform a final check based on the computerized prescription. The concomitant use and interconnection of the three systems will probably be the most powerful way to optimize the medication process and to improve patient safety.

\section{Interests}

Although information technologies are now relatively widely used in hospitals, relatively few robust data exist regarding their impact on the safety of the medication process [19-21]. Exceptions are CPOE and CDSSs, which have been found to improve drug safety. Other previously described innovations, such as automated dispensing devices, scanning and computerization of the MAR, though less studied, should all theoretically have an impact on the error rates.

According to a systematic review [22] on the effect of CPOE on medication safety, at least two well-conducted studies [23][24] demonstrated an important decrease of 55 to $81 \%$ in the serious medication error rate. When the results were evaluated by steps in the process from ordering to administration, it was interesting to consider that CPOE diminished ordering errors by only $19 \%$, whereas transcription $(-84 \%)$, dispensing $(-68 \%)$ and administration $(-59 \%)$ errors were more markedly reduced [25]. The reduction of ordering errors is strongly dependent on the exhaustiveness of the CDSSs associated to the prescription tool.

Although the use of automated dispensing devices results in several benefits, including a net saving in personnel time, an increase of pharmacist availability for clinical activities, and an improvement in billing efficiency [25][26], a reduction in medication errors has not been uniformly realized. We performed an experimental study that demonstrated an abolition of selection errors when drugs where stored in individualized pockets with selective opening ('cubies') connected to an electronic prescription [27], but additional data in real life are needed to confirm this potential. Such results would be very useful to quantify better the impact of automatic dispensing on medication safety.

Bar coding of drugs also seems useful for reducing error rates, although few data from health care are available. Some unpublished data suggest that an $80 \%$ fall in medication administration errors was obtained in an hospital [28]. Another study showed an improvement in error rates for point of care glucose and blood gas testing [29].

To summarize, although the usefulness of information technologies in the reduction of medication error is definite, studies measuring the impact of up-to-date technologies are lacking. The main factors leading to such an improvement are the complete and legible character of the information, the access to structured and powerful clinical decision support, the suppression of transcription steps, the reduction of possibilities for selection error, and the unequivocal identification of drugs and patients.

\section{Barriers}

Although an increased use of IT in the future years seems to be unavoidable, its implantation is a real challenge and numerous experiences have failed. The cultural and organizational changes caused by the introduction of IT must not be underestimated. These tools should only be introduced after a careful estimation of technical (e.g. conviviality, response time, reliability), human (e.g. leadership, value to users, motivation, acceptance, time availability) and ergonomic (e.g. workflow organization) aspects [30], with a special focus on new risks potentially induced by the modification of the process. For CPOEs, a tool assessing hospital readiness was developed [31] including several components such as external environment, organizational leadership, structure and culture, care standardization, order management, access to information, information technology structure and infrastructure.

To prescribe a drug with an electronic tool takes more time than to write orders on paper [32-34]. To make this extra time acceptable to physicians, the clinical decision support system must be powerful and bring significant added value to the prescription. The use of an electronic prescribing system can also induce new risks [35] that are strongly dependent on the system used. Computer entry errors (e.g. wrong drug, wrong dosage, wrong patient) are probably a significant problem, as they were the fourth leading cause of error spontaneously reported in 2003 to the US pharmacopoeia reporting system [36].

The potential limitations of automated dispensing systems are a variety of process deviations, including nurses waiting at busy administration times, especially if there are not enough machines, removal of doses ahead of time to circumvent waiting, and overriding the device when a dose is needed quickly [37].

The efficacy of scanning technology is linked to the presence of an electronic identification on drugs, patients, and care- 
givers. The bar-coding of drug unit doses is not common in the pharmaceutical industry, which would force repackaging by the pharmacy to apply this technology. In the USA, the FDA now require barcodes on the unitdoses of all drugs [38] and it can be hoped that this problem will be mostly resolved in the future years. Even if the reliability of scanning is much higher than human ability, these technologies still do not achieve zero errors, as operators continue to utilize manual data entry in some situations (e.g. scan unsuccessful or unavailable) and patients can wear an erroneous wristband [29].

Last but not least, IT technologies are expensive [39] and the financial aspect often limits implementation. Savings in medication error management and time are probably greater in most, though not all, instances, but IT requires a large up-front capital investment that is often difficult to obtain from hospital managers.

\section{Conclusion}

IT has a huge potential to help reduce the occurrence of medication error, but hospitals need to consider the implications these systems will have on workflow before complete implementation occurs [40]. Factors reported as being important for success or failure are: technical features, user-friendliness, organization of the implementation, and cultural and behavioral patterns of hospital personnel [41][42]. Re-engineering of the medication process should be done using a system approach, evaluating the consecutive steps (prescription, dispensation, administration) and trying to develop a coherent information system connecting them together.

In the Geneva university hospitals, a 2200-bed university hospital, an institutional project of electronic integrated patient records including CPOE and CDSS is on the way. CPOE is actually implemented for nearly 800 beds and evaluation studies are under discussion. We are also applying IT on pilot processes, e.g. chemotherapy, to try to better understand the interests and potential problems of these technologies from the prescription (CPOE) to the administration (scanning). In this case, the implementation is coupled with a systematic risk analysis method (FMECA).

Even if IT constitutes an important way to prevent medication errors, other actions such as healthcare worker education, centralized intravenous additive services (CIVAS) and clinical pharmacy are complementary approaches that should not be forgotten. Ideally, personnel time saved during IT implementation, if any, should be redirected to these activities [43].
[1] Institute of Medicine, 'To err is human. Building a safer health system', Washington DC, National Academy Press, 1999.

[2] T. Brennan, L. Leape, N. Laird, N. Engl. J. Med. 1991, 324, 370.

[3] E. Thomas, D. Studdert, J. Newhouse, Inquiry 1999, 36, 255.

[4] D. Phillips, N. Christenfeld, L. Glynn, Lancet 1998, 351, 643.

[5] D.W. Bates, D. Cullen, N. Laird, JAMA 1995, 274, 29.

[6] T. Hassall, C. Daniels, Am. J. Hosp. Pharm. 1983, 40, 970.

[7] B. Meier, P. Garnerin, P. Chopard, R. Muller, P. Bonnabry, $6^{\text {th }}$ Congress of the European Association of Hospital Pharmacists, Amsterdam, 2001 (abstract).

[8] T. Bayne, R. Bindler, J. Nurs. Staff. Dev. 1997, 13, 293.

[9] J. Reason, $B M J$ 2000, 320, 768.

[10] T. Buchanan, K. Barker, J. Gibson, B. Jiang, R. Pearson, Am. J. Hosp. Pharm. 1991, 48, 2137.

[11] E. Flynn, K. Barker, J. Gibson, R. Pearson, L. Smith, B. Berger, Hum. Factors 1996, $38,614$.

[12] E. Flynn, K. Barker, J. Gibson, R. Pearson, B. Berger, L. Smith, Am. J. Health Syst. Pharm. 1999, 56, 1319.

[13] L. Leape, D.W. Bates, D. Cullen, JAMA 1995, 274, 35 .

[14] H. Guchelaar, M. Kalmeijer, Pharm. World Sci. 2003, 25, 83.

[15] J. Ash, P. Gorman, W. Hersch, Proc. AMIA Symp. 1998, 235.

[16] C. Pedersen, P. Schneider, J. Santell, Am. J. Health Syst. Pharm. 2001, 58, 2251.

[17] A. Darby, Am. J. Health Syst. Pharm. 1996, 53, 1128.

[18] C. Pedersen, P. Schneider, D. Scheckelhoff, Am. J. Health Syst. Pharm. 2003, 60, 52.

[19] E. Oren, E. Shaffer, B. Guglielmo, Am. J. Health Syst. Pharm. 2003, 60, 1447.

[20] D.W. Bates, BMJ 2000, 320, 788.

[21] D.W. Bates, A. Gawande, N. Engl. J. Med. 2003, 348, 2526.

[22] R. Kaushal, K. Shojania, D.W. Bates, Arch. Intern. Med. 2003, 163, 1409.

[23] D.W. Bates, L. Leape, D. Cullen, JAMA 1998, 280, 1311.

[24] D.W. Bates, J. Teich, J. Lee, J. Am. Med. Inform. Assoc. 1999, 6, 313.

[25] M. Ray, L. Aldrich, P. Lew, Hosp. Pharm. 1995, 30, 18.

[26] H. Schwarz, B. Brodowy, Am. J. Health Syst. Pharm. 1995, 52, 823.

[27] P. Bonnabry, C. Du Pasquier, L. Riberdy, P. Garnerin, P. Chopard, 9th Congress of the European Association of Hospital Pharmacists, 2004 (in abstract of the congress).

[28] R. Kaushal, D.W. Bates, Qual. Saf. Health Care 2002, 11, 261.

[29] J. Nichols, C. Bartholomew, M. Brunton, C. Cintron, S. Elliott, J. Mc Girr, D. Morsi, S. Scott, J. Seipel, D. Sinha, Clin. Leadersh. Manag. Rev. 2004, 18, 328.

[30] J. Ash, P. Stavri, G. Kuperman, J. Am. Med. Inform. Assoc. 2003, 10, 229.
[31] D. Stablein, E. Welebob, E. Johnson, J. Metzger, R. Burgess, D. Classen, Jt Comm. J. Qual. Saf. 2003, 29, 336.

[32] W. Hersch, JAMA 2002, 288, 1955.

[33] M. Kalmeijer, W. Holtzer, R. van Dongen, H. Guchelaar, Pharm. World Sci. 2003, 25 , 88.

[34] K. Shu, D. Boyle, C. Spurr, Medinfo 2001, 10, 1207.

[35] R. Koppel, J. Metlay, A. Cohen, B. Abaluck, A. Localio, S. Kimmel, B. Strom. JAMA 2005, 293, 1197.

[36] A. Robeznieks, AMNews, January 24, 2005 (www.amednews.com).

[37] J. Borel, K. Rascati, Am. J. Health Syst. Pharm. 1995, 52, 1875.

[38] Food and Drug Administration Press Office, Press release, February 25, 2004 (http://www.fda.gov/bbs/topics/ news/2004/hhs_022504.html, accessed 2005 Jan 28).

[39] G. Kuperman, R. Gibson, Ann. Intern. Med. 2003, 139, 31 .

[40] D.W. Bates, M. Cohen, L. Leape, J. Overhage, M. Shabot, T. Sheridan, J. Am. Med. Inform. Assoc. 2001, 8, 299.

[41] A. Ahmad, P. Teater, T. Bentley, J. Am. Med. Inform. Assoc. 2002, 9, 16.

[42] J. Ash, P. Stavri, R. Dykstra, L. Fournier, Int. J. Med. Inform. 2003, 69, 235.

[43] J. Carmenates, M. Keith, Am. J. Health Syst. Pharm. 2001, 58, 779. 